\title{
Prognostic ability of DNA-binding protein inhibitor ID-1 expression in patients with oral squamous cell carcinoma
}

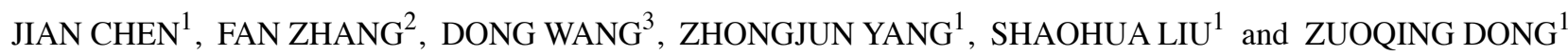 \\ ${ }^{1}$ Department of Oral and Maxillofacial Surgery, Qilu Hospital, Shandong University; ${ }^{2}$ Department of Orthodontics, \\ School and Hospital of Stomatology, Shandong University Qilu Hospital, Shandong University, Jinan, Shandong 250012; \\ ${ }^{3}$ Department of Oral and Maxillofacial Surgery, Qilu Hospital of Shandong University, Qingdao, Shandong 266035, P.R. China
}

Received September 8, 2019; Accepted March 2, 2020

DOI: $10.3892 / \mathrm{ol} .2020 .11506$

\begin{abstract}
DNA-binding protein inhibitor ID-1 (ID-1) plays a vital role in the development of cancer. In the present study, ID-1 expression in oral squamous cell carcinoma (OSCC), and its association with prognosis were investigated in 128 patients with OSCC, treated at the Qilu Hospital of Shandong University and followed up for an additional 10 years. Immunohistochemical analysis was performed to detect ID-1 expression, and the association between ID-1 expression and recurrence, and estimated disease-specific survival (DSS) time were subsequently analyzed using the Mann-Whitney $\mathrm{U}$ test and the Kaplan-Meier method, respectively. In addition, the log-rank test was implemented to compare the survival curves and multivariate Cox proportional hazards analysis was performed to assess the prognostic value of ID-1. The results demonstrated that ID-1 was highly expressed in the majority of OSCC tissues investigated, and ID-1 expression was significantly higher in cases with recurrence of local tumors and lymph node metastasis. Furthermore, higher ID-1 expression levels were associated with a shorter DSS time. Taken together, the results of the present study suggest that ID-1 may serve as an independent prognostic factor to predict DSS time in patients with OSCC.
\end{abstract}

\section{Introduction}

Oral squamous cell carcinoma (OSCC) is characterized as squamous cell carcinoma originated from lips, buccal mucosa, anterior tongue, floor of the mouth, hard palate, gingiva and retromolar trigone (1). It is the eighth most fatal malignancy worldwide, accounting for $3.01 \%$ of all cancer cases and $1.79 \%$ of cancer-associated mortalities in 2019 in the United

Correspondence to: Dr Zuoqing Dong, Department of Oral and Maxillofacial Surgery, Qilu Hospital, Shandong University, 107 West Wenhua Road, Jinan, Shandong 250012, P.R. China

E-mail: dongzq@126.com

Key words: oral squamous cell carcinoma, inhibitor of differentiation/DNA binding-1, prognosis, survival analysis
States (2,3). Despite considerable progress in clinical treatment, the overall 5-year survival rate of OSCC was $\sim 60 \%$ in United States in 2005 to 2011 (4). Tumor recurrence and metastasis are considered the most common causes of mortality with OSCC (5). A number of tumor suppressor genes and oncogenes, including DNA-binding protein inhibitor ID-1 (ID-1) are currently used to predict the prognosis of different types of malignant tumor. For example, the expression levels of ID-1 were correlated with poor prognosis in epithelial ovarian tumors, nasopharyngeal carcinoma and head and neck squamous cell carcinoma (6-8).

ID-1 plays a vital role in the development of cancer and is associated with poor patient outcome (9). ID-1 predominantly elevated in mRNA and protein levels in several types of cancer, such as prostate, breast and ovarian cancer (10), contributing to tumorigenesis by inhibiting cellular differentiation, stimulating proliferation and facilitating tumor neoangiogenesis (10). A previous study demonstrated that overexpression of ID-1 protein was associated with tumor angiogenesis in OSCC; however, due to a short follow-up time, the association between ID-1 expression and survival rate was unable to be determined (11).

To the best of our knowledge, few studies have investigated the association between ID-1 expression and survival rate in OSCC (11-13). Thus, the present study analyzed long-term follow-up data to determine the association between ID-1 expression and survival rate, and establish its role as a potential prognostic marker for patients with OSCC.

\section{Materials and methods}

Patient information and follow-up data. The present research was a retrospective study. A total of 128 patients with OSCC, who underwent tumor resection and neck dissection at the Qilu Hospital of Shandong University (Shandong, China) were enrolled into the present study from January 2004 to October 2008. The inclusion criterion was OSCC without distant metastasis that the tumor had not undergone distant metastasis. A total of 128 oral cancer tissues and 60 adjacent normal tissues were collected, and the margin of non-neoplastic tissues was $1-1.5 \mathrm{~cm}$ away from tumor edge with biopsy-negative. The surgical procedure selected the range of neck dissection according to the primary lesions 
and metastatic lymph nodes in the neck of the patient. Supraomohyoid neck dissection was used to select patients in clinical stage I, while radical neck dissection (RND) or modified RND (in which the sternocleidomastoid muscle, internal jugular vein, spinal accessory nerve were retained) were used to select patients in clinical stages II and III. The clinical stages of OSCC were classified according to the American Joint Committee on Cancer staging manual (14). Patients with primary lesions $>4 \mathrm{~cm}$ in length or with cervical lymphatic metastasis received postoperative radiotherapy. The patients were in stage II or III of OSCC. There were total 67 patients. The treatment was 2 Gy per day duration for one month with a total dose of $60 \mathrm{~Gy}$. The clinicopathological characteristics of the patients with OCSS are presented in Table I. Excluding those lost to follow-up, each patient was followed-up for 10 years, until October 2018. Disease-specific survival (DSS) time was considered to be the interval between surgery and time of mortality due to OSCC. Data were censored for patients who died from other causes (such as heart disease, chronic lower respiratory disease or diabetes mellitus) or at the time of the last follow-up. The deaths directly caused by OSCC were counted as death cases, while deaths caused by other reasons or lost to follow-up during treatment were not included. Other causes of death included heart disease, chronic lower respiratory diseases and diabetes mellitus etc.

Immunohistochemistry (IHC). IHC was performed on 128 oral cancer tissues and 60 adjacent normal tissues (the margin of tissues with biopsy-negative, $1-1.5 \mathrm{~cm}$ away from tumor edge). There were just 60 adjacent normal tissues, so 60 adjacent normal tissues were assessed. Paraffin-embedded sample sections (4- $\mu \mathrm{m}$ thickness) were dewaxed and rehydrated in xylene and graded ethanol $(100,95,90,70 \%$ and each grade for $5 \mathrm{~min})$. The slides were then subjected to antigen retrieval using $0.01 \mathrm{M}$ citric buffer ( $\mathrm{pH}$ 6.0) for $20 \mathrm{~min}$, followed by incubation in $3 \% \mathrm{H}_{2} \mathrm{O}_{2}$ at room temperature for $10 \mathrm{~min}$. Non-specific binding was blocked by incubation in $10 \%$ goat serum for $10 \mathrm{~min}$ at room temperature. Slides were then washed and incubated with primary antibodies of ID-1 (rabbit polyclonal antibody, cat. no. PA5-101010, dilution 1:400, Santa Cruz Biotechnology, Inc.) at $4^{\circ} \mathrm{C}$ overnight. The next day, the slides were washed in phosphate-buffered saline (PBS) three rinses (each for $5 \mathrm{~min}$ ) and incubated with multimer anti-rabbit/mouse horseradish peroxidase(HPR)-conjugated secondary antibodies (cat. no. SV0004, dilution 1:300, Wuhan Boster Biological Technology, Ltd.) for $30 \mathrm{~min}$ at $37^{\circ} \mathrm{C}$. Then slides were washed and visualized by adding the substrate DAB. The slides were then rinsed in distilled water and counterstained with haematoxylin for $3 \mathrm{~min}$ at room temperature. The slides were observed, and images were captured using a light phase-contrast microscope at x400 magnification (Leica, Microsystems). Immunoreactivity was graded based on the density and degree of positively-stained cells (15). Patients were subsequently divided into two groups, according to the ID-1 expression level median value: Low expression group (absent/weak, <3.7) and high expression group (median/strong, >3.7).

Prognostic indicators. The difference in ID-1 expression was compared between patients with and without tumor recurrence. Univariate survival analysis was performed to assess the association between ID-1 expression and survival rate, while multivariate analysis was performed to determine whether ID-1 may act as an independent prognostic factor for DSS time in patients with OSCC. Previous research showed that there was significant correlation between ID-1 expression levels and tumor size, clinical stage, lymph node involvement (11). Pathologic differentiation grade has also been shown to be an important indicator of clinical prognosis in squamous cell carcinoma (16). Therefore, in multivariate survival analysis, the following characteristics were assessed according to previously established grading systems $(11,16)$ : Tumor size, pathological differentiation grade, clinical stage, lymph node metastasis and ID-1 expression. The overall survival rate which was calculated through the total survival cases/total cases. Pathological differentiation grades were classified as follows: Well, well-differentiated; moderate, moderately differentiated; poor, poorly differentiated (17). Clinical stage were classified as follows: Stage I, T1N0M0; Stage II, T2N0M0; Stage III, T3N0M0, T(1-3)N1M0; Stage IV, T4aN(0,1)M0, T(1-4a)N2M0, TN3M0 and T4bNM0 (14). Lymph node metastasis was classified as negative or positive.

Statistical analysis. Statistical analysis was performed using SPSS software (version 23.0; IBM Corp). Data are presented as mean \pm standard deviation. An unpaired t-test was used to compare the expression of ID-1 in nonneoplastic oral tissues and OSCC. The Mann-Whitney test was used to assess the association between ID-1 expression and recurrence. The Kaplan-Meier method was used to assess DSS times, while the log-rank test was implemented to compare survival curves. Five and ten-year survival descriptive data are presented of the cases, and no further statistical analysis was performed on these data. Multivariate Cox proportional hazards analysis was performed to determine the prognostic value of ID-1. $\mathrm{P}<0.05$ was considered to indicate a statistically significant difference.

\section{Results}

Follow-up parameters. Follow-up data were received from all patients, with a median survival time of $68.00 \pm 14.89$ months (range, 4-120 months). Compared with the previous statistics (11), there were 65 new cases of local recurrence and 34 new cases of lymph node metastasis. A total of 93 patients $(72.7 \%)$ died during the follow-up period, of which $83(64.8 \%)$ died of OSCC and $10(7.8 \%)$ died of other causes, such as heart disease, chronic lower respiratory diseases and diabetes mellitus. A further 19 patients (14.8\%) were lost to follow-up, thus only 16 patients (12.5\%) survived the entire follow-up period. These data are presented in Table I.

ID-1 expression in OSCC tissues. ID-1 expression was significantly higher in OSCC tissues compared with that in nonneoplastic oral tissues $(\mathrm{P}<0.0001$; Fig. 1). Among the 60 adjacent normal tissues, ID-1 expression was absent in 36 cases and weakly expressed in 24 cases. Among the 128 OSCC tissues, cytoplasmic and nuclear ID-1 protein expression level was negative in 21 cases (Fig. 2A), and 24 cases exhibited weak ID-1 expression levels, with weak nuclear staining and partial cytoplasmic expression (Fig. 2B). Moderate expression levels of ID-1 were exhibited in 64 cases, 
Table I. Clinicopathological characteristics of patients with oral squamous cell carcinoma $(n=128)$.

\begin{tabular}{|c|c|}
\hline Characteristic & Patient, n $(\%$ \\
\hline \multicolumn{2}{|l|}{ Age, years } \\
\hline$\leq 60$ & $68(53.1)$ \\
\hline$>60$ & $60(46.9)$ \\
\hline \multicolumn{2}{|l|}{ Sex } \\
\hline Male & $90(70.3)$ \\
\hline Female & $38(29.7)$ \\
\hline \multicolumn{2}{|c|}{ Tumor size, mm } \\
\hline$<31$ & $65(50.8)$ \\
\hline$\geq 31$ & $63(49.2)$ \\
\hline \multicolumn{2}{|l|}{ Tumor location } \\
\hline Tongue & $45(35.2)$ \\
\hline Gingival & $29(22.7)$ \\
\hline Mouth floor & $16(12.5)$ \\
\hline Lip & $10(7.8)$ \\
\hline Cheek & $16(12.5)$ \\
\hline Soft palate & $12(9.4)$ \\
\hline \multicolumn{2}{|c|}{ Pathological differentiation grade } \\
\hline Well & $74(57.8)$ \\
\hline Moderate & $42(32.8)$ \\
\hline Poor & $12(9.4)$ \\
\hline \multicolumn{2}{|l|}{ Clinical stage } \\
\hline $\mathrm{I}+\mathrm{II}$ & $71(55.5)$ \\
\hline III+IV & $57(44.5)$ \\
\hline
\end{tabular}

Pathological differentiation grade: Well, Well-differentiated; Moderate, Moderately differentiated; Poor, Poorly differentiated. Clinical stage: Stage I, T1NOM0; Stage II, T2NOM0; Stage III, T3NOM0, T(1-3) N1M0; Stage IV, T4aN(0,1)M0, T(1-4a)N2M0, TN3M0, T4bNM0.

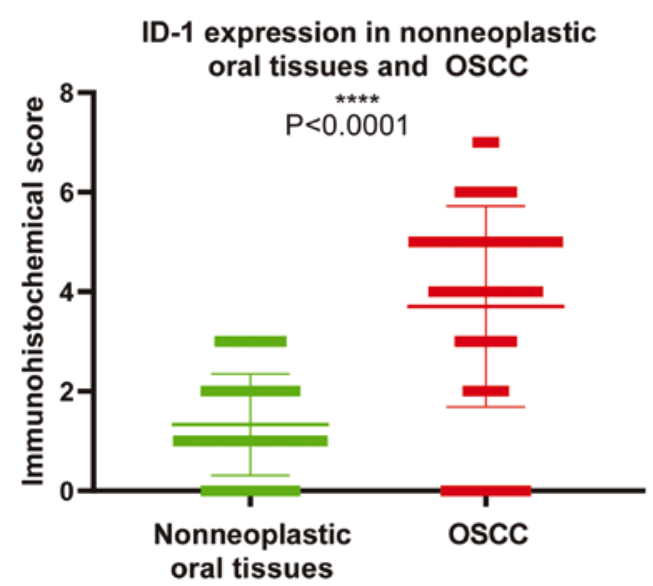

Figure 1. ID-1 expression and immunohistochemical score between OSCC and nonneoplastic oral tissue samples. ID-1, DNA-binding protein inhibitor ID-1; OSCC, oral squamous cell carcinoma.

with areas of intense staining in the cytoplasm and nucleus (Fig. 2C). Strong ID-1 expression was observed in 19 cases, where the majority of the cytoplasm and nucleus were

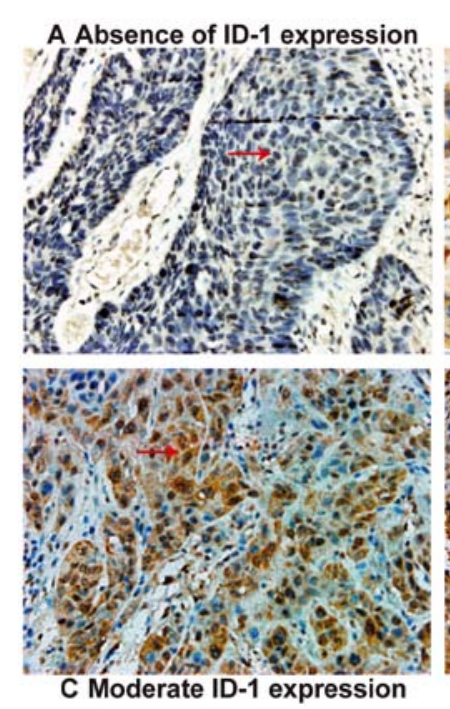

B Weak ID-1 expression

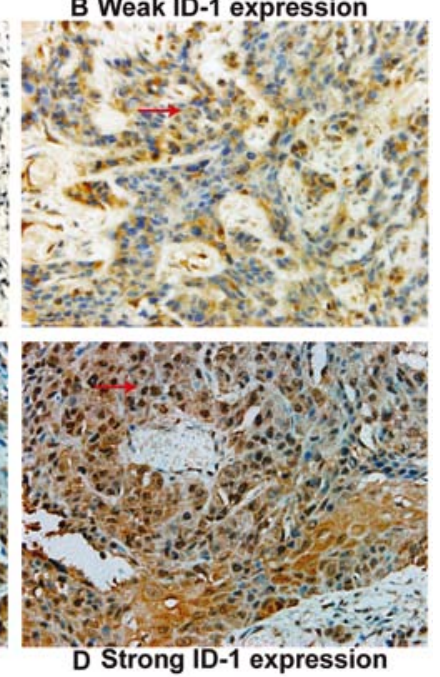

Figure 2. ID-1 expression in oral squamous cell carcinoma tissues. (A) Negative ID-1 expression in the cytoplasm and nucleus. (B) Weak ID-1 expression, with little nuclear and partial cytoplasmic staining. (C) Moderate ID-1 expression, with areas of intense staining in the cytoplasm and nucleus. (D) Strong ID-1 expression, with majority of areas positively stained in the cytoplasm and nucleus. Magnification, x400. ID-1, DNA-binding protein inhibitor ID-1.

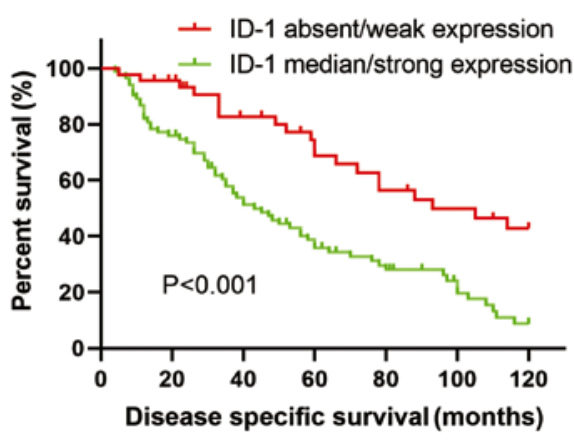

Figure 3. Disease-specific survival time in patients with oral squamous cell carcinoma, according to ID-1 expression levels. Patients in the high ID-1 expression level group had significantly shorter DSS times compared with those with low ID-1 expression levels. ID-1, DNA-binding protein inhibitor ID-1.

positively stained (Fig. 2D). The low ID-1 expression group (absent/weak) consisted of 45 cases and the high ID-1 expression group (median/strong) consisted of 83 cases.

High ID-1 expression is associated with tumor recurrence. ID-1 expression was significantly higher in patients with local tumor and lymph node metastasis recurrence compared with those without recurrence, $4.01 \pm 1.92$ vs. $2.92 \pm 2.06(\mathrm{P}=0.007)$ and $4.08 \pm 1.90$ vs. $2.98 \pm 2.06(\mathrm{P}=0.001)$, respectively (Table II).

High ID-1 expression is associated with poor survival. The median DSS times in the low and high ID-1 protein expression

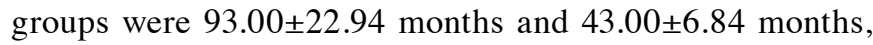
respectively. Patients with higher ID-1 protein expression levels exhibited significantly shorter DSS times compared with those with lower ID-1 expression levels ( $\mathrm{P}<0.001$; Table III). The 5- and 10-year disease-specific survival curve (univariate survival analysis) is shown in Fig. 3. The 5- and 10-year 
Table II. Associations between ID-1 expression levels and recurrence in patients with oral squamous cell carcinoma.

\begin{tabular}{lcc}
\hline Classification & Patient, $n(\%)$ & ID-1 expression (mean \pm SD) \\
\hline Local tumor recurrence & & \\
Negative & $36(28.1)$ & $2.92 \pm 2.06$ \\
Positive & $92(71.9)$ & $4.01 \pm 1.92$ \\
Lymph node metastasis recurrence & & \\
Negative & $44(34.4)$ & $2.98 \pm 2.06$ \\
Positive & $84(65.6)$ & $4.08 \pm 1.90$ \\
Total & $128(100.0)$ & $3.67 \pm 1.94$ \\
\hline
\end{tabular}

${ }^{a}$ Mann Whitney test. DNA-binding protein inhibitor ID-1; SD, standard deviation.

Table III. Kaplan-Meier survival analysis in patients with oral squamous cell carcinoma, according to ID-1 expression.

\begin{tabular}{lclc}
\hline & & \multicolumn{2}{c}{ Disease-specific survival, months } \\
\cline { 3 - 4 } Classification & Patient number, $\mathrm{n}$ & Mean \pm SD & Median \pm SD \\
\hline Absent/weak ID-1 expression & 45 & $86.08 \pm 6.02$ & $93.00 \pm 22.94$ \\
Median/strong ID-1 expression & 83 & $54.97 \pm 4.43$ & $43.00 \pm 6.84$ \\
Total & 128 & $70.53 \pm 5.23$ & $68.00 \pm 14.89$ \\
\hline
\end{tabular}

${ }^{a}$ Log-rank test. ID-1, DNA-binding protein inhibitor ID-1.

Table IV. Survival rates in patients with oral squamous cell carcinoma.

\begin{tabular}{lcc}
\hline Classification & $\begin{array}{c}\text { 5-year } \\
\text { survival } \\
\text { rate, } \%\end{array}$ & $\begin{array}{c}\text { 10-year } \\
\text { survival } \\
\text { rate, \% }\end{array}$ \\
\hline Absent/weak ID-1 expression & 75 & 55 \\
Median/strong ID-1 expression & 40 & 23 \\
Total & 52 & 34 \\
\hline
\end{tabular}

ID-1, DNA-binding protein inhibitor ID-1.

overall survival rates between the two groups are presented in Table IV.

ID-1 is an independent prognostic factor for DSS time in patients with OSCC. Multivariate survival analysis identified that ID-1 protein expression levels $(\mathrm{P}=0.002)$ and lymph node metastasis $(\mathrm{P}=0.034)$ are independent prognostic factors for DSS time in patients with OSCC (Table V).

\section{Discussion}

ID-1 plays a number of key roles in cellular differentiation, cell cycle progression, tumorigenesis and metastasis (18). ID-1 predominantly acts as an oncogene in several types of tumor, including gastric (19), pancreatic (20), colorectal (21) and lung cancer (22). Furthermore, a previous study demonstrated that
Table V. Multivariate survival analysis for disease-specific survival in patients with oral squamous cell carcinoma.

\begin{tabular}{lccc}
\hline Characteristic & $95 \%$ CI & OR & P-value $^{\mathrm{a}}$ \\
\hline Tumor size & $0.99-1.04$ & 1.102 & 0.394 \\
Pathologic differentiation grade & $0.94-1.82$ & 1.305 & 0.114 \\
Clinical stage & $0.99-2.12$ & 1.464 & 0.054 \\
Lymph node metastasis & $1.04-2.93$ & 1.750 & 0.034 \\
ID-1 expression & $1.08-1.42$ & 1.239 & 0.002 \\
\hline
\end{tabular}

${ }^{a}$ Cox's proportional hazards regression analysis. CI, confidence interval; OR, odds ratio.

higher protein levels of ID-1 are associated with tumor recurrence and lymph node metastasis in patients with OSCC (11). However, the association between ID-1 expression levels and the survival status of postoperative patients has not investigated. To the best of our knowledge, the present study was the first to analyze long-time follow-up data, to investigate the association between ID-1 expression levels and survival rates in patients with OSCC.

The present study identified 65 new cases of local tumor recurrence and 34 new cases of recurring lymph node metastasis, and the results demonstrated a significant association between ID-1 expression levels and tumor recurrence. These results were consistent with those of a previous study (11), which provides support that high ID-1 expression levels are associated with poor prognosis in patients with OSCC, thus 
suggesting that ID-1 may serve as an indicator of patient outcome.

The results of the present study were also consistent with a previous study on oral squamous cell carcinoma demonstrating that high ID-1 expression levels were associated with poor prognosis (12). However, the results from a recent report on primary esophageal squamous cell carcinoma (ESCC) contradicted the results in the present study, suggesting that strong ID-1 expression levels in the metastatic lymph nodes from patients with ESCC were associated with improved prognosis (23). Conversely, another study on ESCC reported that ID-1 was a predictor of poor patient survival (24). A reason for this discrepancy may be due to the fact that metastases results from the survival and proliferation of primary tumor cells, tumor evolution and progression, which can result in biological diversity between different patients (25). A number of previous studies have demonstrated that some gene expression profiles and biomarkers between primary and metastatic tumors are differentially expressed, such as programmed cell death protein-1 and zeste homolog-2 (26-28). In renal cell carcinoma, the expression levels ofPD-1, programmed cell death-ligand 1 (PD-L1) and PD-L2 between primary and metastatic sites are different (26). Tissue microarray and cDNA microarray analysis revealed differential gene and protein expression levels in primary breast tumors and corresponding lymph node metastases (27). In metastatic breast cancer, the expression levels of the zeste homolog-2, estrogen receptor, progesterone receptor and human epidermal growth factor receptor 2 were different between primary and metastatic lesions (28). In the present study, ID-1 expression was not detected in metastatic lymph nodes. The comparison of ID-1 expression between the primary tumor and metastatic lesion in the same case is more convincing. Thus, investigations into the differential expression pattern of ID-1 in metastatic paired lymph nodes, and the association between ID-1 and prognosis should be investigated in prospective studies.

Despite improvements in therapeutic strategies, the overall 5 -year survival rate of OSCC was $\sim 60 \%$ in United States between 2005 and 2011 (4). The results of the present study indicated that the 5-year survival rate of OSCC was 52\%, while the 10 -year survival rate was $34 \%$. This is consistent with the results of an epidemiological survey on OSCC (29). Local tumor recurrence and lymph node metastasis are considered the primary factors contributing to poor prognosis and low 5-year survival rates of patients with OSCC (30). Thus, long-term patient follow-up was investigated in the present study, and ID-1 expression level was demonstrated to be associated with survival rate. Univariate survival analysis indicated that the median DSS times of patients in the low ID-1 expression level group were significantly longer compared with those with high ID-1 expression levels. Furthermore, multivariate analysis demonstrated that ID-1 expression levels and lymph node metastasis were independent prognostic factors for DSS time in patients with OSCC, which was consistent with findings from previous studies on different types of tumors, such as non-small cell lung cancer and glioblastoma $(22,31)$. In non-small cell lung cancer, high ID-1 expression levels were associated with a shorter survival time (22), and genetic knockdown of ID-1 led to a significant increase in survival time in a nude mice with glioblastoma (31).
The molecular mechanism by which ID-1 promotes tumor recurrence and lymph node metastasis has not yet been fully investigated. It is hypothesized that ID-1 may promote lymphangiogenesis by upregulating the expression of vascular endothelial growth factor C in OSCC (32). Previous studies have reported that ID-1 interacts with different signaling molecules and pathways, such as the PI3K/AKT and Wnt/ $\beta$-Catenin signaling pathways, which regulate the proliferation, migration, metastasis and chemoresistance of the tumor (18). ID-1 activated the NF- $\mathrm{NB}$ signaling pathway to promote migration and invasion in non-small cell lung cancer cells (22).

MicroRNAs (miRNAs) have also been reported to play a key role in the regulation of ID1 expression. Through targeting bone morphogenetic protein receptor type 1A and blocking BMP/Smad/ID-1 signaling, miRNA-885-3p was demonstrated to inhibit growth of HT-29 colon cancer cell xenografts in nude mice via angiogenetic disruption (33). These previous studies laid the foundation for clinical and mechanistic studies of ID-1 in OSCC; however, further research is required to fully understand its role therein.

The present study was not without limitations. First, the present study was a retrospective study with a single center cohort, which comprised a limited heterogeneous sample group. Furthermore, the effect of postoperative radiotherapy was not considered. The limitations might have a bias effect on the results; however, the present study featured long-term follow-up of 128 patients, therefore the results should be reliable and reproducible.

In conclusion, the present study demonstrated that high ID-1 expression levels were significantly associated with local tumor recurrence and lymph node metastasis, suggesting that ID-1 may serve as an independent prognostic factor for DSS time in patients with OSCC. An initial assessment of the impact of ID-1 on patients with OSCC has been provided; however, validation analysis of a large-scale prospective homogeneous sample cohort is still required to fully determine the role of ID-1 in OSCC.

\section{Acknowledgements}

Not applicable.

\section{Funding}

The present study was funded by the Key Technology Research and Development Program of Shandong, China (grant no. 2017GSF221008).

\section{Availability of data and materials}

The datasets used and/or analyzed during the present study are available from the corresponding author upon reasonable request.

\section{Authors' contributions}

JC designed the present study and wrote the manuscript. DW acquired the data. FZ contributed to data analysis and interpretation. ZY contributed to the data collection and analysis. SL participated in drafting the initial manuscript 
and performed statistical analysis. ZD designed the study and revised the manuscript. All authors have read and approved the final manuscript.

\section{Ethics approval and consent to participate}

The present study was approved by The Ethics Committee of Qilu Hospital of Shandong University (Jinan, China) and informed consent was provided by all the patients prior to the start of the study.

\section{Patient consent for publication}

Not applicable.

\section{Competing interests}

The authors declare that they have no competing interests.

\section{References}

1. Chow LQM: Head and neck cancer. N Engl J Med 382: 60-72, 2020.

2. Siegel RL, Miller KD and Jemal A: Cancer statistics, 2019. CA Cancer J Clin 69: 7-34, 2019.

3. Shield KD, Ferlay J, Jemal A, Sankaranarayanan R, Chaturvedi AK, Bray F and Soerjomataram I: The global incidence of lip, oral cavity, and pharyngeal cancers by subsite in 2012. CA Cancer J Clin 67: 51-64, 2017.

4. Siegel RL, Miller KD and Jemal A: Cancer statistics, 2016. CA Cancer J Clin 66: 7-30, 2016.

5. Chinn SB and Myers JN: Oral cavity carcinoma: Current management, controversies, and future directions. J Clin Oncol 33: 3269-3276, 2015.

6. Schindl M, Schoppmann SF, Ströbel T, Heinzl H, Leisser C, Horvat R and Birner P: Level of Id-1 protein expression correlates with poor differentiation, enhanced malignant potential, and more aggressive clinical behavior of epithelial ovarian tumors. Clin Cancer Res 9: 779-785, 2003.

7. Sun W, Guo MM, Han P, Lin JZ, Liang FY, Tan GM, Li HB, Zeng $M$ and Huang XM: Id-1 and the p65 subunit of NF- $\kappa B$ promote migration of nasopharyngeal carcinoma cells and are correlated with poor prognosis. Carcinogenesis 33: 810-817, 2012.

8. Lin J, Guan Z, Wang C, Feng L, Zheng Y, Caicedo E, Bearth E, Peng JR, Gaffney P and Ondrey FG: Inhibitor of differentiation 1 contributes to head and neck squamous cell carcinoma survival via the NF-kappaB/survivin and phosphoinositide 3-kinase/Akt signaling pathways. Clin Cancer Res 16: 77-87, 2010.

9. Benezra R, Davis RL, Lockshon D, Turner DL and Weintraub H: The protein Id: A negative regulator of helix-loop-helix DNA binding proteins. Cell 61: 49-59, 1990.

10. Perk J, Iavarone A and Benezra R: Id family of helix-loop-helix proteins in cancer. Cancer 5: 603-614, 2005.

11. Dong Z, Liu S, Zhou C, Sumida T, Hamakawa H, Chen Z, Liu P and Wei F: Overexpression of Id-1 is associated with tumor angiogenesis and poor clinical outcome in oral squamous cell carcinoma. Oral Oncol 46: 154-157, 2010.

12. Nishimine M, Nakamura M, Mishima K, Kishi M, Kirita T, Sugimura M and Konishi N: Id proteins are overexpressed in human oral squamous cell carcinomas. J Oral Pathol Med 32: 350-357, 2003

13. Zhao J, Wang S, Liu N and Tang X: Correlation between the expression of Id-1 and hyperthermia-associated molecules in oral squamous cell carcinoma. J Clin Pathol 66: 758-763, 2013.

14. Lydiatt WM, Patel SG, O'Sullivan B, Brandwein MS, Ridge JA, Migliacci JC, Loomis AM and Shah JP: Head and neck cancers-major changes in the American joint committee on cancer eighth edition cancer staging manual. CA Cancer J Clin 67: 122-137, 2017.

15. Schoppmann SF, Schindl M, Bayer G, Aumayr K, Dienes J, Horvat R, Rudas M, Gnant M, Jakesz R and Birner P: Overexpression of Id-1 is associated with poor clinical outcome in node negative breast cancer. Int J Cancer 104: 677-682, 2003.
16. Kyrgidis A, Tzellos TG, Kechagias N, Patrikidou A, Xirou P, Kitikidou K, Bourlidou E, Vahtsevanos K and Antoniades K: Cutaneous squamous cell carcinoma (SCC) of the head and neck: Risk factors of overall and recurrence-free survival. Eur J Cancer 46: 1563-1572, 2010.

17. Wu JM and Montgomery E: Classification and pathology. Surg Clin North Am 88: 483-520, 2008.

18. Ke J, Wu R, Chen Y and Abba ML: Inhibitor of DNA binding proteins: Implications in human cancer progression and metastasis. Am J Transl Res 10: 3887-3910, 2018.

19. Li L, Wei X, Wu B, Xiao Y, Yin M and Yang Q: siRNA-mediated knockdown of ID1 disrupts Nanog- and Oct-4-mediated cancer stem cell-likeness and resistance to chemotherapy in gastric cancer cells. Oncol Lett 13: 3014-3024, 2017.

20. Georgiadou D, Sergentanis TN, Sakellariou S, Filippakis GM, Zagouri F, Vlachodimitropoulos D, Psaltopoulou T, Lazaris AC, Patsouris E and Zografos GC: VEGF and Id-1 in pancreatic adenocarcinoma: Prognostic significance and impact on angiogenesis. Eur J Surg Oncol 40: 1331-1337, 2014.

21. Zhao ZR, Zhang ZY, Zhang H, Jiang L, Wang MW and Sun XF: Overexpression of Id-1 protein is a marker in colorectal cancer progression. Oncol Rep 19: 419-424, 2008.

22. Li J, Li Y, Wang B, Ma Y and Chen P: Id-1 promotes migration and invasion of non-small cell lung cancer cells through activating NF- $\kappa$ B signaling pathway. J Biomed Sci 24: 95, 2017.

23. Hu Y, Luo KJ, Wen J and Zhu ZH: Strong expression of Id-1 in metastatic lymph nodes from esophageal squamous cell carcinoma is associated with better clinical outcome. J Thorac Dis 10: 5499-5507, 2018

24. Yuen HF, Chan YP, Chan KK, Chu YY, Wong ML, Law SY, Srivastava G, Wong YC, Wang X and Chan KW: Id-1 and Id-2 are markers for metastasis and prognosis in oesophageal squamous cell carcinoma. Br J Cancer 97: 1409-1415, 2007.

25. Fidler IJ and Hart IR: Biological diversity in metastatic neoplasms: Origins and implications. Science 217: 998-1003, 1982.

26. Zhang X, Yin X, Zhang H, Sun G, Yang Y, Chen J, Zhu X, Zhao P, Zhao J, Liu J, et al: Differential expressions of PD-1, PD-L1 and PD-L2 between primary and metastatic sites in renal cell carcinoma. BMC Cancer 19: 360, 2019.

27. Hao X, Sun B, Hu L, Lähdesmäki H, Dunmire V, Feng Y, Zhang SW, Wang $\mathrm{H}$, Wu C, Wang $\mathrm{H}$, et al: Differential gene and protein expression in primary breast malignancies and their lymph node metastases as revealed by combined cDNA microarray and tissue microarray analysis. Cancer 100: 1110-1122, 2004.

28. Inari H, Suganuma N, Kawachi K, Yoshida T, Yamanaka T, Nakamura Y, Yoshihara M, Nakayama H, Yamanaka A, Masudo K, et al: Expression of enhancer of zeste homolog 2 correlates with survival outcome in patients with metastatic breast cancer: Exploratory study using primary and paired metastatic lesions. BMC Cancer 17: 160, 2017.

29. Patel SG, Amit M, Yen TC, Liao CT, Chaturvedi P, Agarwal JP, Kowalski LP, Ebrahimi A, Clark JR, Cernea CR, et al: Lymph node density in oral cavity cancer: Results of the international consortium for outcomes research. Br J Cancer 109: 2087-2095, 2013.

30. Tabatabaeifar S, Larsen MJ, Larsen SR, Kruse TA, Thomassen M and Sørensen JA: Investigating a case of possible field cancerization in oral squamous cell carcinoma by the use of next-generation sequencing. Oral Oncol 68: 74-80, 2017.

31. Soroceanu L, Murase R, Limbad C, Singer E, Allison J, Adrados I, Kawamura R, Pakdel A, Fukuyo Y, Nguyen D, et al: Id-1 is a key transcriptional regulator of glioblastoma aggressiveness and a novel therapeutic target. Cancer Res 73: 1559-1569, 2013.

32. Dong Z, Wei F, Zhou C, Sumida T, Hamakawa H, Hu Y and Liu S: Silencing Id-1 inhibits lymphangiogenesis through down-regulation of VEGF-C in oral squamous cell carcinoma. Oral Oncol 47: 27-32, 2011.

33. Xiao F, Qiu H, Cui H, Ni X, Li J, Liao W, Lu L and Ding K: MicroRNA-885-3p inhibits the growth of HT-29 colon cancer cell xenografts by disrupting angiogenesis via targeting BMPR1A and blocking BMP/Smad/Id1 signaling. Oncogene 34: 1968-1978, 2015.

This work is licensed under a Creative Commons Attribution-NonCommercial-NoDerivatives 4.0 International (CC BY-NC-ND 4.0) License. 